\title{
BMJ Open Association between depressive symptoms and arterial stiffness: a cross- sectional study in the general Chinese population
}

Liming Peng, Sisi Bi, Xiangwei Liu, Tianyi Long, Yixia Zhao, Fei Li, Tianlun Yang, Chenglong Zhang (D)

To cite: Peng L, Bi S, Liu X, et al. Association between depressive symptoms and arterial stiffness: a crosssectional study in the general Chinese population. BMJ Open 2020;10:e033408. doi:10.1136/ bmjopen-2019-033408

- Prepublication history and additional material for this paper are available online. To view these files, please visit the journal online (http://dx.doi. org/10.1136/bmjopen-2019033408).

Received 03 August 2019 Revised 20 January 2020 Accepted 11 February 2020

Check for updates

(C) Author(s) (or their employer(s)) 2020. Re-use permitted under CC BY-NC. No commercial re-use. See rights and permissions. Published by BMJ.

Department of Cardiology, Xiangya Hospital, Central South University, Changsha, Hunan, China

Correspondence to Dr Chenglong Zhang; chenglongzhang@csu.edu.cn

\section{ABSTRACT}

Objectives To determine the independent relationship between depressive symptoms and arterial stiffness in the general Chinese population, and to explore possible interactive factors in the relationship.

Design A cross-sectional study.

Setting and participants Consecutive participants who received routine health physical examination in an affiliated hospital of a comprehensive university in Hunan Province, China, between September 2013 and March 2014 were examined. After exclusion of subjects not meeting the criteria, a total of 1334 subjects aged 22-77 years were recruited for final analysis.

Measures The Patient Health Questionnaire-9 was employed to assess the degree of depressive symptoms: 0-4 no depressive symptoms, 5-9 mild depressive symptoms and 10-27 moderate to severe depressive symptoms. Brachial-ankle pulse wave velocity (baPWV) was measured to determine arterial stiffness.

Results There was a slight increase in baPWV across elevated degrees of depressive symptoms ( $p=0.025)$. Multivariate linear regression analysis revealed that mild depressive symptoms and moderate to severe depressive symptoms were independently associated with baPWV compared with no depressive symptoms after adjusting for baseline confounders (beta-coefficient: 40.3, 95\% Cl 6.6 to 74.1; beta-coefficient: $87.7,95 \% \mathrm{Cl} 24.0$ to 151.5 , respectively). Further stratified analyses indicated that the relationship between degree of depressive symptoms and baPWV was predominant in subjects who had normal or normal-high blood pressure, or combined with hypertension ( $p$ for interaction $=0.016$ ), or in subjects with diabetes mellitus ( $p$ for interaction $=0.004$ ), examined in multivariate linear regressions. In addition, after adjustment, a significant association between moderate to severe depressive symptoms and baPWW was also found in female subjects younger than 60 years, although the interactive effect was not significant ( $p$ for interaction $=0.056$ ). Conclusions Depressive symptoms are independently associated with arterial stiffness, especially in subjects whose blood pressures are beyond the optimal range and combined with diabetes mellitus.

\section{INTRODUCTION}

Major depressive disorder (MDD) is one of the most common psychological disorders
Strengths and limitations of this study

- This study analysed the association between depressive symptoms and arterial stiffness in the general Chinese population covering a wide range of ages (22-77 years).

- The extent of depression was reflected by mild depressive symptoms and moderate to severe depressive symptoms, and the independent relationship of these indicators with brachial-ankle pulse wave velocity was examined in multivariate linear regression models.

- Various subgroup analyses were conducted to explore whether any interactive factors existed in the relationship between depressive symptoms and arterial stiffness.

- Diagnostic interviews according to the criteria of the Diagnostic and Statistical Manual of Mental Disorders, Fourth Edition, were not performed to obtain a diagnosis of major depressive disorder in subjects with moderate to severe depressive symptoms.

- Due to the cross-sectional design of the study, no clear cause-effect conclusion could be directly drawn.

that affect health-related quality of life. ${ }^{1}$ The global prevalence of MDD is $4.7 \%$, and its lifetime rate varies greatly across different races, cultures and regions, ranging from $3.3 \%$ in mainland China to $18.6 \%$ in the USA. $^{2-4}$ Furthermore, the prevalence of MDD in patients with cardiovascular disease (CVD) is much higher ${ }^{5}: 26.8 \%$ in subjects with hypertension, ${ }^{6} 21.5 \%$ in patients with heart failure $^{7}$ and $20.0 \%$ in patients with acute coronary syndrome (ACS) ${ }^{8}$ In addition, MDD was demonstrated to be an independent risk factor for poor prognosis in patients with ACS. ${ }^{8}{ }^{9}$ It was estimated that almost two-thirds of middle-aged and older adults with depression also reported a diagnosis of comorbid CVD. ${ }^{10}$ Therefore, there 
exist manifold interrelations between MDD and CVD where both contribute to a poor prognosis. ${ }^{5}$

Arterial stiffness can reflect arterial elasticity and the burden of arteriosclerosis and atherosclerosis. ${ }^{11}$ Pulse wave velocity (PWV) is regarded as the gold standard measurement of large artery stiffness and is one of the markers of hypertension-mediated organ damage, and should be assessed among patients with hypertension according to the guidelines of the European Society of Hypertension (ESH) and the European Society of Cardiology (ESC). ${ }^{12}$ Previous meta-analyses have revealed that PWV was an independent predictor of the development of CVD, adverse cardiovascular events and all-cause mortality. ${ }^{13-15}$ At present, PWV is extensively applied in both clinical practice and epidemiological studies based on its feasibility and clinical significance.

Large population-based studies on the relationship between depression and arterial stiffness are limited, and the results remain controversial. The Rotterdam Study $(\mathrm{n}=3704, \geq 60$ years $)$ and the Age, Gene/Environment Susceptibility-Reykjavik Study (AGES-Reykjavik Study) $(\mathrm{n}=2058$, mean age 79.6 \pm 4.6 years) reported that both depressive symptoms and major depression were associated with aortic stiffness reflected by carotid-femoral PWV (cfPWV). ${ }^{16}{ }^{17}$ The association between the severity of depressive symptoms and arterial stiffness reflected by cfPWV and the augmentation index was also verified in another two studies with small sample sizes which recruited adolescents $(n=157$, aged 16-21 years) and patients with depressive and/or anxiety disorder $(n=449$, aged 20-66 years), respectively. ${ }^{18} 19$ The Maastricht Study $(\mathrm{n}=2757$, aged $40-75$ years $)$ indicated that the independent associations of depressive symptoms and MDD with cfPWV were restricted among middle-aged men (aged 40-60 years). ${ }^{20}$ Furthermore, the Health, Aging, and Body Composition Study ( $\mathrm{n}=2488$, aged $70-79$ years) failed to establish a link between depressive symptoms and $c f P W V .{ }^{21}$ Finally, the Netherlands Study of Depression and Anxiety ( $\mathrm{n}=635$, aged 20-66 years) also failed to identify an association between depression sensitivity and central arterial stiffness assessed by the augmentation index. ${ }^{22}$

The main reasons for the abovementioned diverse findings might be differences in the enrolled population, assessment methods of arterial stiffness and criteria for defining depression. We observed that most studies mainly focused on middle-aged and older participants, and none of the studies included subjects with a wide range of ages. In view of these findings, we selected a general population without a specific age restriction and aimed to test the relationship between depressive symptoms assessed by the Patient Health Questionnaire-9 (PHQ-9) and arterial stiffness reflected by brachial-ankle PWV (baPWV). Additionally, we explored whether the association (if present) differed among subgroups according to various baseline factors; this type of analysis was seldom performed in previous studies.

\section{METHODS}

\section{Study subjects}

The current study followed a cross-sectional design and recruited a general Chinese population. We collected the medical information of consecutive participants who received routine health physical examination voluntarily at the Health Management Center of Xiangya Hospital, Central South University between September 2013 and March 2014. Subjects meeting any of the following criteria were excluded: age $<18$ or $\geq 80$ years; history of myocardial infarction, heart failure, stroke, cancer, and severe hepatic or renal dysfunction; serious mental disorders, such as schizophrenia, bipolar disorder and schizoaffective disorder; any missing data from PHQ-9 or baPWV; or unwillingness to participate in the survey. A total of 1632 patients were included during the entry period, and after further exclusion 1334 participants (860 men and 474 women, mean age $47.1 \pm 11.7$ years) were finally analysed in this study.

\section{Data collection}

Participants' basic information was collected by experienced trained medical staff at the Health Management Center according to relevant standard procedures. All subjects were asked about their cigarette smoking status and medical history of hypertension and diabetes mellitus. Height was measured to the nearest $0.1 \mathrm{~cm}$ with the participants wearing no shoes, and weight was measured to the nearest $0.1 \mathrm{~kg}$ with the participants wearing light indoor clothing and no shoes. Body mass index (BMI) was calculated as weight divided by height squared $\left(\mathrm{kg} / \mathrm{m}^{2}\right)$. Systolic blood pressure (SBP) and diastolic blood pressure (DBP) were measured using a mercury sphygmomanometer with the participants in a seated position after at least $5 \mathrm{~min}$ of rest. The mean of two separate readings of blood pressure with an interval of 3-5 min between measurements was used. Fasting blood samples were collected from the antecubital veins after an 8-hour overnight fast in the morning of the health check-up. Fasting blood glucose (FBG) and lipid profiles, including total cholesterol, triglycerides, highdensity lipoprotein cholesterol (HDL-C) and low-density lipoprotein cholesterol (LDL-C), were measured using an automatic biochemistry analyser (Beckman AU5800, Koutou-ku, Tokyo, Japan) in the central laboratory immediately after obtaining the blood samples.

All participants were classified into three categories based on their blood pressure and according to the ESC and ESH hypertension guidelines ${ }^{12}$ : optimal blood pressure, normal and high-normal blood pressure, and hypertension. An optimal blood pressure was defined as SBP $<120 \mathrm{~mm} \mathrm{Hg}$ and DBP $<80 \mathrm{~mm} \mathrm{Hg}$; a normal and highnormal blood pressure was defined as SBP between 120 and $139 \mathrm{~mm} \mathrm{Hg}$ and/or DBP between 80 and $89 \mathrm{~mm} \mathrm{Hg}$; and hypertension was defined as $\mathrm{SBP} \geq 140 \mathrm{~mm} \mathrm{Hg}$ and/ or DBP $\geq 90 \mathrm{~mm} \mathrm{Hg}$, a self-reported history of diagnosed hypertension, or currently under antihypertensive treatment. Diabetes mellitus was defined as FBG $\geq 7.0 \mathrm{mmol} / \mathrm{L}$, 
Table 1 Baseline characteristics of all subjects according to degree of depressive symptoms

\section{Depressive symptoms}

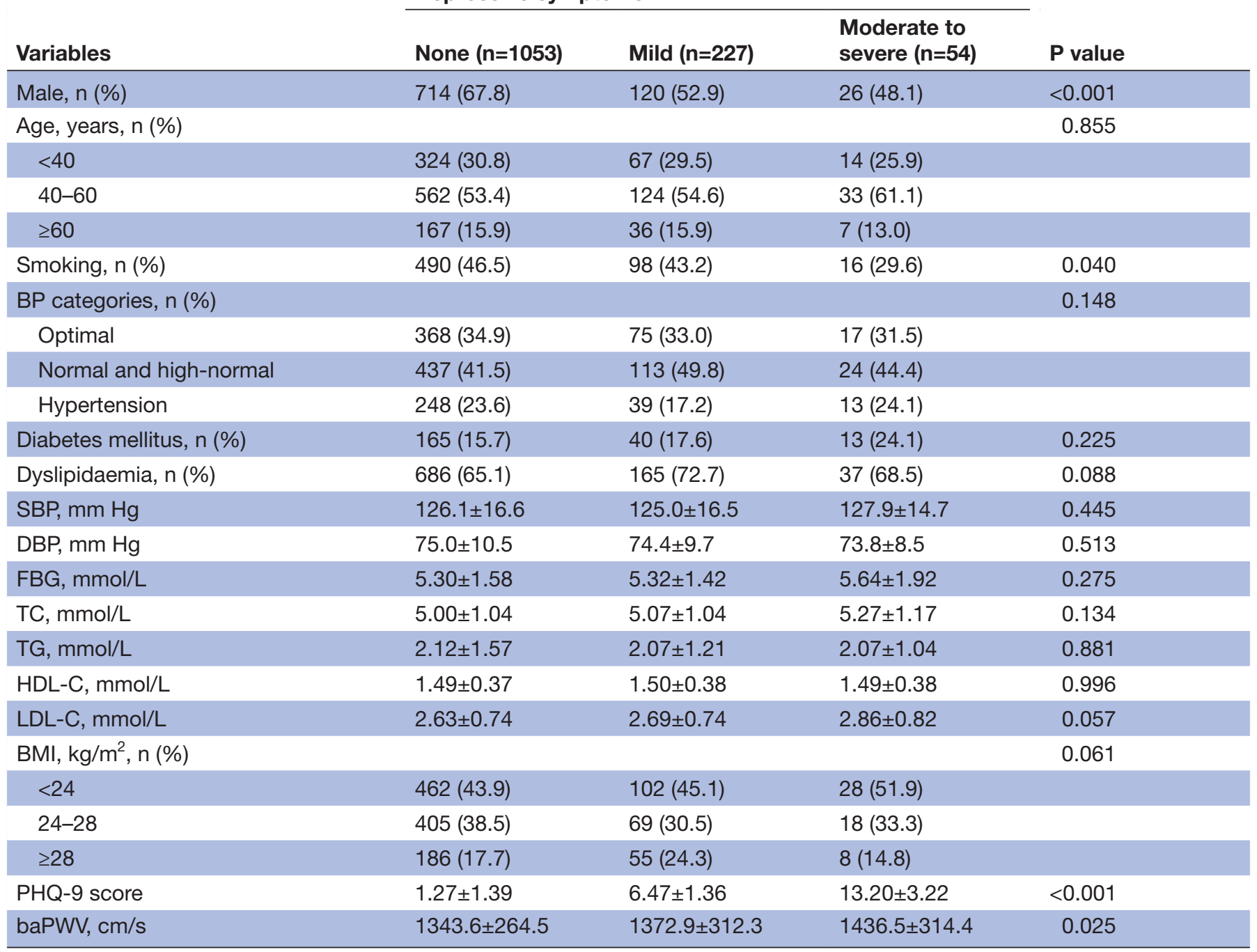

Data are presented as mean $\pm \mathrm{SD}$ or $\mathrm{n}$ (percentage) as appropriate.

baPWV, brachial-ankle pulse wave velocity; BMI, body mass index; BP, blood pressure; DBP, diastolic blood pressure; FBG, fasting blood glucose; HDL-C, high-density lipoprotein cholesterol; LDL-C, low-density lipoprotein cholesterol; PHQ-9, Patient Health Questionnaire-9; SBP, systolic blood pressure; TC, total cholesterol; TG, triglyceride.

a self-reported history of diagnosed diabetes mellitus or currently undergoing hypoglycaemic therapy. The diagnosis of dyslipidaemia was made based on the Korean guidelines for dyslipidaemia in the general population. ${ }^{23}$ BMIs of $24 \mathrm{~kg} / \mathrm{m}^{2}$ and $28 \mathrm{~kg} / \mathrm{m}^{2}$ were used to identify overweight and obesity, respectively, for the Chinese population.

The PHQ-9 is a self-administered questionnaire designed to screen depression in primary care and other settings, and consists of nine items that are each scored from 0 to 3 points depending on the frequency of the listed problems in the last 2 weeks. The total score on PHQ-9 ranges from 0 to 27 , and scores are categorised according to the following: 0-4 no depression, 5-9 mild depression, 10-14 moderate depression, 15-19 moderately severe depression and 20-27 severe depression. ${ }^{24}$ A cut-off value of 10 on PHQ-9 has been widely used in epidemiological studies to diagnose MDD, with high sensitivity (85\%) and specificity $(89 \%) .{ }^{25}$ Referring to previous relevant studies and considering the limited sample size of subjects with a PHQ-9 score $\geq 10 \quad(n=54)$, in the current study we classified the entire population into three groups according to the following PHQ-9 scores: 0-4 no depressive symptoms, 5-9 mild depressive symptoms and 10-27 moderate to severe depressive symptoms.

baPWV was measured using an automatic baPWV instrument (Model BP-203RPE, Colin, Komaki City, Japan) by trained staff following standard procedures, and was measured with participants in supine position after $10 \mathrm{~min}$ of rest in a quiet room with comfortable temperature. Bilateral measurements of baPWV were recorded, and the higher reading was used for analysis. This automatic device and its reproducibility have been previously validated. 
Table 2 Association between depressive symptoms and baPWV in univariate and multivariate linear analyses

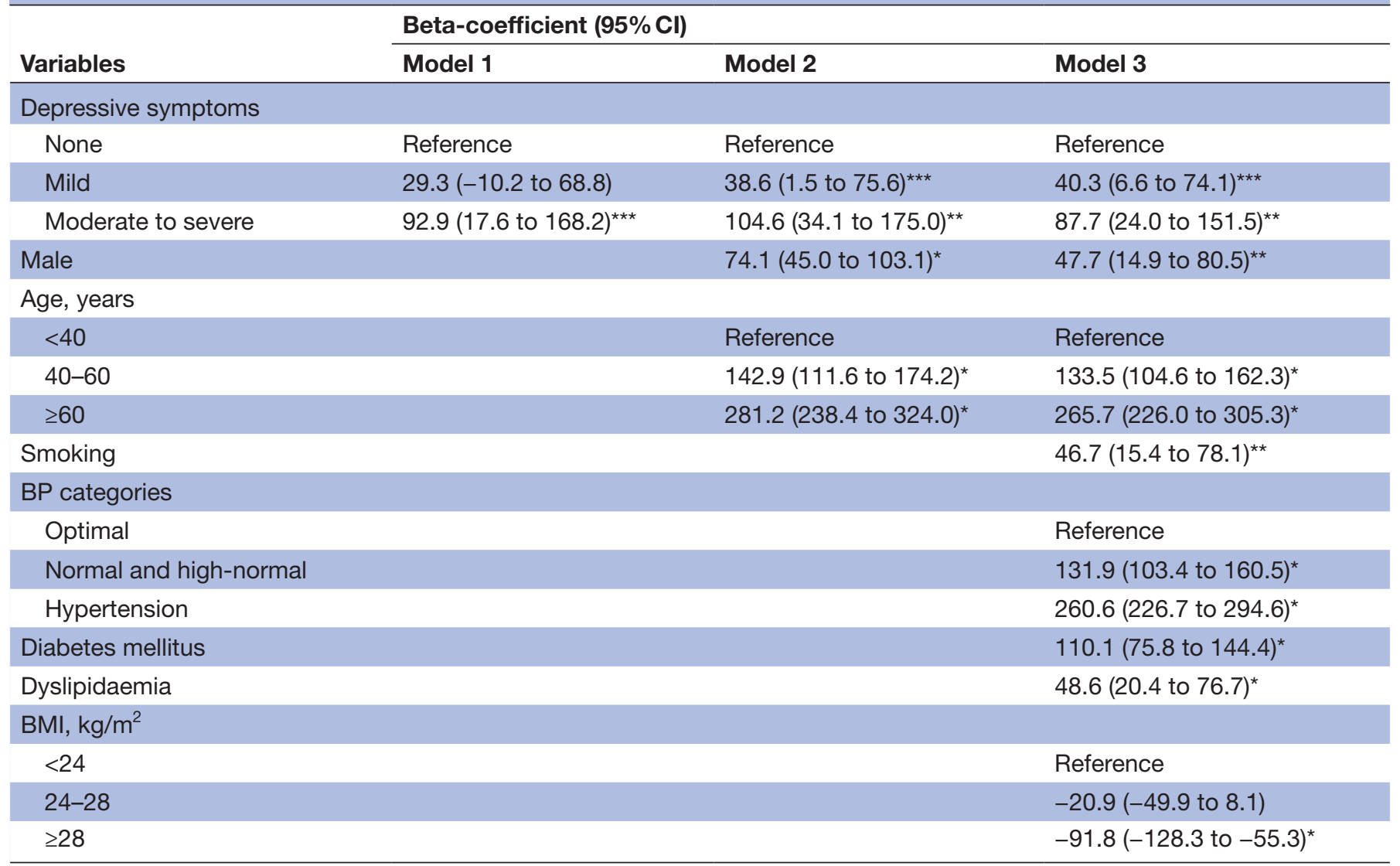

${ }^{*} \mathrm{P}<0.001,{ }^{* *} \mathrm{P}<0.01,{ }^{* *} \mathrm{P}<0.05$.

baPWV, brachial-ankle pulse wave velocity; BMI, body mass index; BP, blood pressure.

\section{Statistical analysis}

Since the association between depression and arterial stiffness has been conflicting and the initial purpose of our data was not for the current study, we did not estimate the sample size needed to obtain significant results. In fact, in the final analysis after exclusion, all baseline characteristics were obtained from the entire population, so there were no missing data in our study. All subjects were divided into three groups according to degree of depressive symptoms as determined by the PHQ-9 score. Continuous variables are presented as mean and SD, while categorical variables are described as frequencies and percentages. Comparisons between groups were performed using analysis of variance for continuous variables and $\chi^{2}$ test for categorical variables. The relationship between degree of depressive symptoms and baPWV was examined in linear regressions with three models. Model 1 was the crude model; model 2 adjusted for sex and age; and model 3 further adjusted for smoking status, blood pressure or BMI, and comorbidities of diabetes mellitus or dyslipidaemia. Stratified analyses of baseline factors, including sex, age, blood pressure or BMI, smoking status, and history of diabetes mellitus or dyslipidaemia, were also performed to test whether any potential interactive effects existed in the relationship between degree of depressive symptoms and baPWV, and each stratification was adjusted for all other factors except the stratification factor itself. The group of no depressive symptoms was considered as reference in the regression models. Finally, we especially explored the joint roles of age and sex in the association between mild and moderate to severe depressive symptoms and baPWV in the adjusted models. All analyses were conducted using statistical software packages R V.3.4.3 (http:/ /www.R-project.org; The R Foundation) and EmpowerStats (www.empowerstats.com; X\&Y Solutions, Boston, Massachusetts). All tests were twotailed, and a p value less than 0.05 was considered statistically significant.

\section{Patient and public involvement}

None of the participants or the public were involved in the study design, data analysis or interpretation of the results of the study.

\section{RESULTS}

\section{Baseline characteristics}

After exclusion of subjects not meeting the criteria, a total of 1334 subjects were included in the final analysis (mean age $41.7 \pm 11.7$ years, $64.5 \%$ male). The detailed baseline characteristics of all subjects by degree of depressive symptoms are described in table 1 . The results indicated there 
Table 3 Association between depressive symptoms and baPWV according to subgroups of baseline characteristics

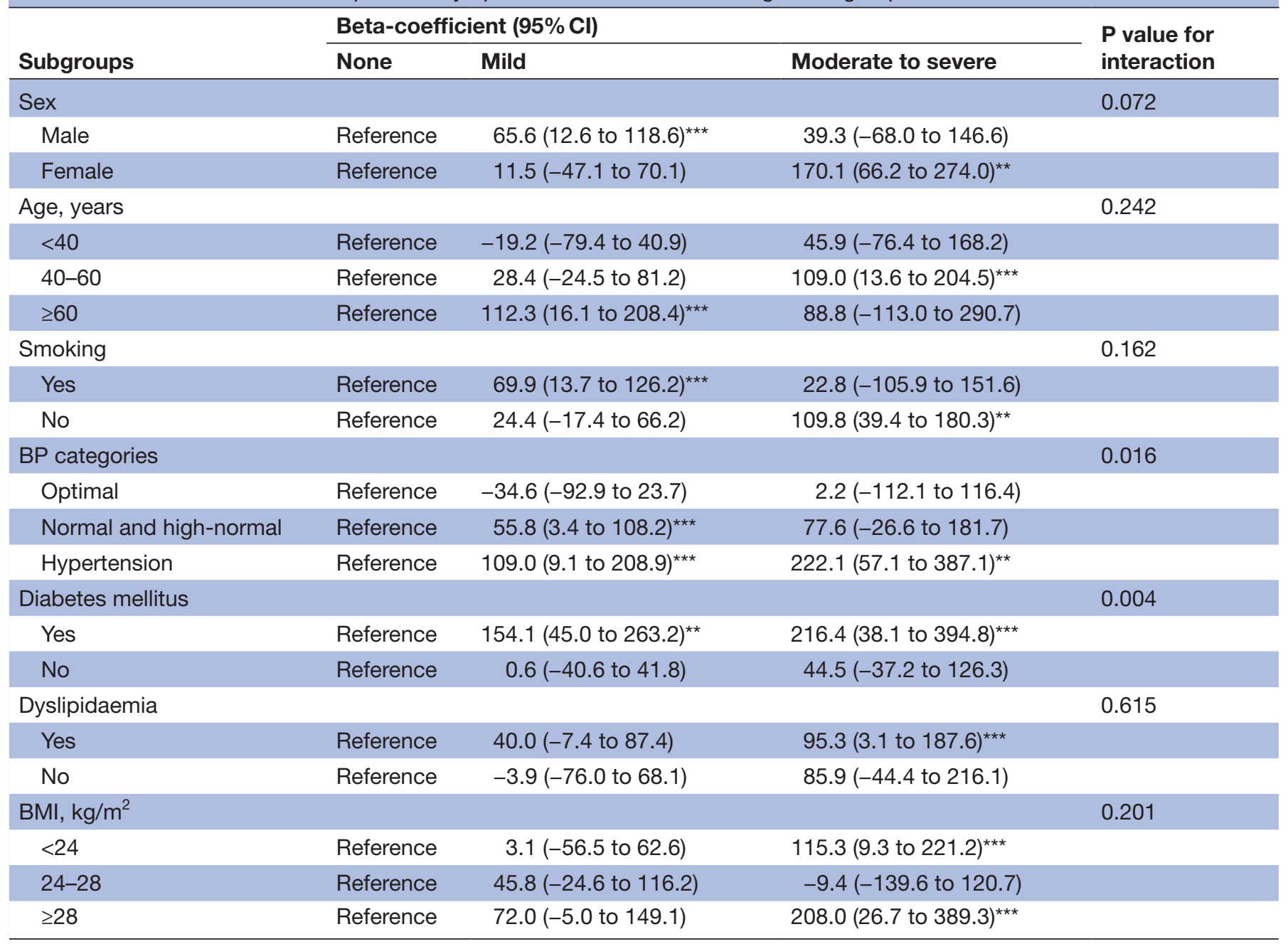

Each stratification was adjusted for all other presented subgroups except the stratification factor itself.

${ }^{*} \mathrm{P}<0.001,{ }^{* *} \mathrm{P}<0.01,{ }^{* * *} \mathrm{P}<0.05$.

baPWV, brachial-ankle pulse wave velocity; BMI, body mass index; BP, blood pressure.

were fewer male participants across increasing degrees of depressive symptoms $(67.8 \%, 52.9 \%$ and $48.1 \%$; $\mathrm{p}<0.001)$, as well as a decreasing proportion of participants with smoking history $(46.5 \%, 43.2 \%$ and $29.6 \%$; $\mathrm{p}=0.040$ ). There was no significant difference in terms of age or age categories, comorbidities of diabetes mellitus or dyslipidaemia, blood pressure categories, BMI or BMI categories, levels of SBP or DBP, FBG, or lipid profiles. As expected, a higher baPWV was found in subjects with more severe depressive symptoms $(1343.6 \pm 264.4 \mathrm{~cm} / \mathrm{s}$, $1372.9 \pm 312.3 \mathrm{~cm} / \mathrm{s}$ and $1436.5 \pm 314.4 \mathrm{~cm} / \mathrm{s} ; \mathrm{p}=0.025)$. The baseline characteristics of all subjects according to tertiles of baPWV $(<1203 \mathrm{~cm} / \mathrm{s}, 1203-1430 \mathrm{~cm} / \mathrm{s}, \geq 1430$ $\mathrm{cm} / \mathrm{s}$ ) are presented in online supplementary table 1 . The results showed that across increasing tertiles of baPWV, there were more subjects who were male, aged $\geq 60$ years, had smoking history, hypertension, diabetes mellitus, dyslipidaemia and moderate to severe depressive symptoms. The levels of SBP, DBP, FBG, total cholesterol and LDL-C and PHQ-9 score also increased with increasing tertiles of baPWV, while the proportion of subjects whose BMI $\geq 28 \mathrm{~kg} / \mathrm{m}^{2}$ decreased with increasing tertiles. There were no significant differences in terms of triglyceride and HDL-C among the three groups.

\section{Depressive symptoms and baPWV}

We assessed the association between degree of depressive symptoms and baPWV in linear regression models, and the beta-coefficients and $95 \%$ CI of both crude and adjusted models are shown in table 2 , where subjects with no depressive symptoms were considered as reference. In crude model 1, a significant association between moderate to severe depressive symptoms and baPWV was detected, while the results were non-significant for mild depressive symptoms and baPWV. In model 2, which adjusted for sex and age, significant results were observed for both mild depressive symptoms and moderate to severe depressive symptoms and baPWV. The results remained significant in model 3, which further adjusted for smoking status, blood pressure, diabetes mellitus, dyslipidaemia and 


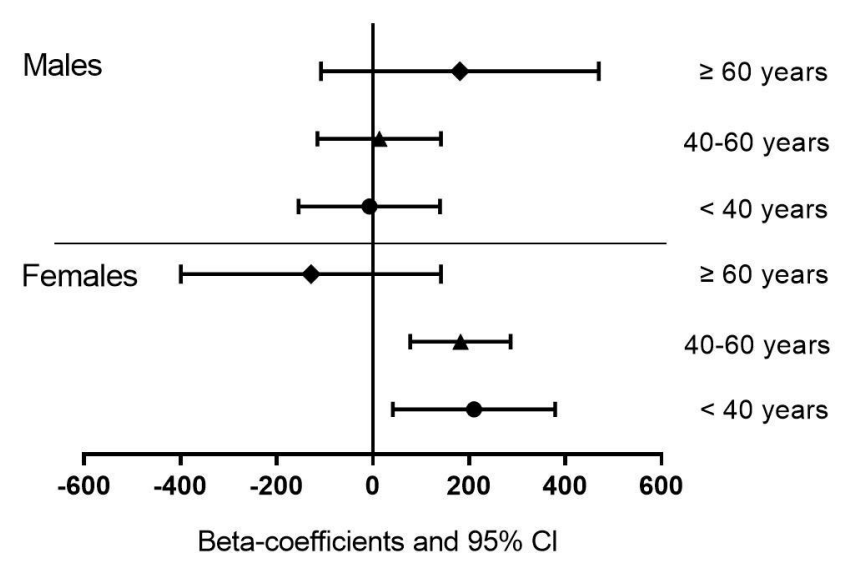

Figure 1 Beta-coefficients and $95 \% \mathrm{Cl}$ of the association between mild depressive symptoms and brachial-ankle pulse wave velocity stratified by age and sex. The results were adjusted for smoking history, blood pressure, diabetes mellitus, dyslipidaemia and body mass index.

BMI (beta-coefficient: 40.3, 95\% CI 6.6 to 74.1 ; betacoefficient: $87.7,95 \%$ CI 24.0 to 151.5 , for mild depressive symptoms and moderate to severe depressive symptoms, respectively).

\section{Subgroup analysis of baseline factors}

We also analysed the association between degree of depressive symptoms and baPWV in subgroups of sex, age, smoking status, blood pressure, diabetes mellitus, dyslipidaemia and BMI, and the results are shown in table 3, with the group with no depressive symptoms still considered as reference. In the stratified analysis of blood pressure, significant associations between mild depressive symptoms and baPWV were obtained in the subgroups of normal and high-normal blood pressure and hypertension (beta-coefficient: 55.8, 95\% CI 3.4 to 108.2; beta-coefficient: $109.0,95 \%$ CI 9.1 to 208.9 , respectively), while a significant association between moderate to severe depressive symptoms and baPWV was observed

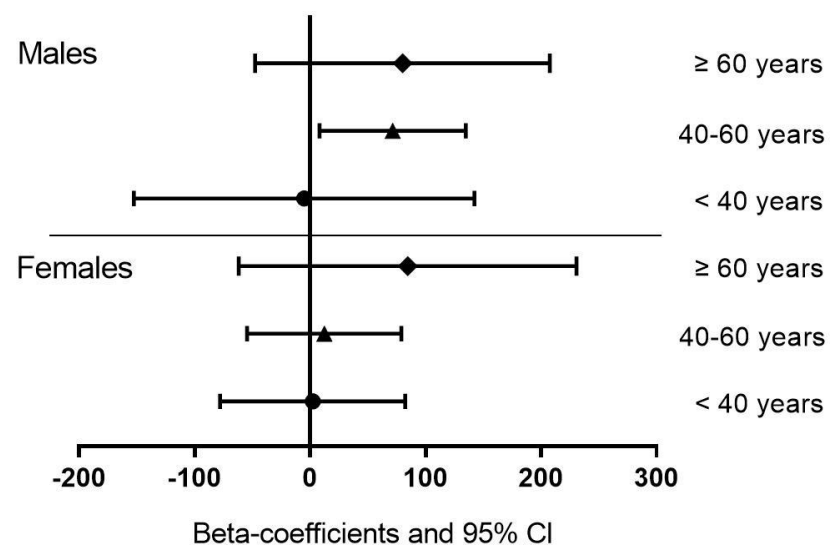

Figure 2 Beta-coefficients and $95 \% \mathrm{Cl}$ of the association between moderate to severe depressive symptoms and brachial-ankle pulse wave velocity stratified by age and sex. The results were adjusted for smoking history, blood pressure, diabetes mellitus, dyslipidaemia and body mass index. only in the hypertension subgroup (beta-coefficient: 222.1, 95\% CI 57.1 to 387.1), with the interaction also significant ( $p$ for interaction $=0.016$ ). In the subgroups of diabetes mellitus, significant associations between mild depressive symptoms and moderate to severe depressive symptoms and baPWV were found in those with diabetes mellitus (beta-coefficient: 154.1, 95\% CI 45.0 to 263.2; beta-coefficient: 216.4, 95\% CI 38.1 to 394.8 ) and the interaction was also significant ( $\mathrm{p}$ for interaction $=0.004$ ). For other subgroups of sex, age, smoking status, dyslipidaemia and BMI, no significant interactions were found ( $p$ for interaction $>0.05$ for all).

\section{Subgroups of age and sex}

The beta-coefficients and the 95\% CI of the association between mild depressive symptoms and baPWV according to age and sex are described in figure 1. Smoking history, blood pressure, diabetes mellitus, dyslipidaemia and BMI were adjusted in the regression models. The results showed that a significant association between mild depressive symptoms and baPWV was observed in male subjects aged 40-60 years (beta-coefficient: 71.6, 95\% CI 8.2 to 135.0), but the result of interaction was not significant ( $p$ for interaction $=0.260$ ). The association between moderate to severe depressive symptoms and baPWV stratified by age and sex is presented in figure 2. There were significant associations between moderate to severe depressive symptoms and baPWV in female subjects younger than 40 years (beta-coefficient: 210.9 , 95\% CI 40.5 to 381.3 ) and aged 40-60 years (beta-coefficient: $185.2,95 \%$ CI 79.5 to 290.9) after adjusting for the same variables in figure 1 , although the result of interaction was not significant ( $p$ for interaction $=0.056$ ).

\section{DISCUSSION}

In the current cross-sectional study, we established an independent association between depressive symptoms and arterial stiffness in the general Chinese population. Subgroup analyses indicated that the associations between degree of depressive symptoms and arterial stiffness were most important in those whose blood pressures were not within the optimal range (including normal, high-normal and diagnosed hypertension) and in those combined with diabetes mellitus. In addition, significant associations between mild depressive symptoms and moderate to severe depressive symptoms and arterial stiffness were observed in male subjects aged 40-60 years and in female subjects younger than 60 years, respectively, in the stratified analysis of age and sex. However, the interactive effects were not significant for both.

The association between depressive symptoms and arterial stiffness in our study was in accordance with the findings of previous cross-sectional investigations. ${ }^{16-19}$ However, the Health, Aging, and Body Composition Study failed to establish an association between depressive symptoms and arterial stiffness as reflected by cfPWV. ${ }^{21}$ In addition, it was remarkable that the Maastricht Study 
revealed that age and sex jointly influenced the associations between depressive symptoms, MDD and arterial stiffness as examined by cfPWV; significant associations existed only in the middle-aged male subgroup (aged $40-60$ years).$^{20}$ In our study, no significant interactive effects were found for the subgroups of sex or age separately in the associations between depressive symptoms and baPWV. We further stratified all subjects by age and sex concurrently to examine whether the associations differed according to age and sex. Contrary to the findings of the Maastricht Study, our results indicated that significant associations between moderate to severe depressive symptoms and arterial stiffness were present only in women who were younger than 60 years, although the interactive effect was not statistically significant. The association between mild depressive symptoms and arterial stiffness was significant only in male subjects aged 40-60 years, which has not been reported before.

The prevalence of MDD in women largely exceeds that in men, a difference that has been reported worldwide. ${ }^{26}$ This sex difference was also exhibited in specific depressive symptoms; for instance, women with depression tended to gain weight than men. ${ }^{27}$ Moreover, an age-sex interaction was found in regard to an increase in carotid arterial stiffness. ${ }^{28}$ It is reasonable that sex might have an interactive effect on the relationship between depression and arterial stiffness, but which sex is more vulnerable to the influence of depression on arterial stiffness seems conflicting according to our findings and the results of the Maastricht Study. It should be noted that the cohort of the Maastricht Study especially included individuals with diabetes according to their study design. ${ }^{29}$ In the Maastricht Study, the prevalence of diabetes mellitus was $27 \%$ among subjects without depressive disorder and $49 \%$ among participants with $\mathrm{MDD},{ }^{20}$ and these figures were higher than those in other cohorts, including ours (the prevalence of diabetes mellitus was $16.3 \%$ ). Therefore, the large demographic difference might explain the opposite findings, and future studies are needed to elucidate clearly the role of age and sex in the relationship between depression and arterial stiffness.

We also classified the whole population into three subgroups based on blood pressure-optimal blood pressure, hypertension, and the rest which indicated normal or high-normal blood pressure-which was according to the current hypertension guidelines. ${ }^{12}$ A significant interactive effect on the association between degree of depressive symptoms and arterial stiffness was found for blood pressure. One previous study showed that severe arterial stiffness was independently associated with progression of blood pressure (eg, normal blood pressure progressed to either high-normal blood pressure or hypertension; highnormal blood pressure progressed to hypertension). ${ }^{30}$ Another longitudinal investigation revealed that in addition to an SBP $>140 \mathrm{~mm} \mathrm{Hg}$, an SBP between 120 and $139 \mathrm{~mm} \mathrm{Hg}$ was also associated with an increase in PWV compared with the PWV associated with SBP $<120 \mathrm{~mm}$ Hg. ${ }^{31}$ However, longitudinal studies have obtained inconsistent results with regard to the association between hypertension and depression. ${ }^{32}{ }^{33}$ In addition, one study inferred that, among the elderly, subjects with low blood pressure (SBP $<120 \mathrm{~mm} \mathrm{Hg}$ or DBP $<75 \mathrm{~mm} \mathrm{Hg}$ ) were at increased risk of incident depression compared with those with normal blood pressure. ${ }^{32}$ Another study revealed that anxiety or depression at baseline was associated with low blood pressure during follow-up. ${ }^{34}$ The role of blood pressure in the interaction between depressive symptoms and arterial stiffness was first reported in our study, and limited by the cross-sectional study design we could not fully elucidate the causality or the underlying mechanism. However, one plausible explanation is that elevated blood pressure and advanced depressive symptoms might jointly promote the development of arterial stiffness. We also observed that older subjects with depressive symptoms tended to have lower night-time SBP fall, ${ }^{35}$ and subjects with white coat hypertension or masked hypertension examined by ambulatory blood pressure monitoring had greater arterial stiffness. ${ }^{36}$ Older subjects with depression were also associated with increased prevalence of left ventricular hypertrophy, which was independent of blood pressure levels. ${ }^{37}$ All these studies indicated that there existed a more complicated relationship between depression, arterial stiffness and blood pressure, and the measures of blood pressure circadian and target organ damage might provide more information.

Another novel finding of the current study was that diabetes mellitus affected the relationship between depressive symptoms and arterial stiffness. Undoubtedly, diabetes mellitus is a well-known traditional risk factor for CVD and can accelerate progression of arterial stiffness. Meta-analyses have indicated that type 2 diabetes mellitus is a risk factor for new-onset depression, and that depression is also inversely associated with incident type 2 diabetes mellitus. ${ }^{38}{ }^{39}$ However, one recent study implied that the association between type 2 diabetes mellitus and MDD, which was examined at the epidemiological and genetic levels, does not exist, leading to controversial results. ${ }^{40}$ Therefore, whether diabetes mellitus is a reliable interaction factor between depressive symptoms and arterial stiffness still needs further investigation.

The possible underlying mechanisms by which depression influences arterial stiffness include the following aspects: inflammation, endothelial dysfunction, dysregulation of the autonomic nerve system (ANS) and unhealthy behavioural patterns. As a result of psychosocial stressors, poor diet, physical inactivity, obesity and smoking, ${ }^{41}$ a chronic and mild inflammatory response plays a role in the emergence of depression from childhood to adulthood, and a variety of proinflammatory cytokines are involved in this process. ${ }^{42}$ In addition, endothelial dysfunction is independently associated with depressive disorders. ${ }^{43}$ Endothelial damage is modulated by selective serotonin reuptake inhibitor treatment in patients with MDD and in vitro cell models. ${ }^{44}$ Dysregulation of the ANS, especially sympathetic overactivity, and activated proinflammatory cytokines can cause imbalances in the kynurenine 
pathway, leading to MDD. ${ }^{45} 46$ Elevated heart rates and plasma catecholamine levels and low heart rate variability were found in the ANS dysfunction groups and were correlated with increased arterial stiffness. ${ }^{47} 48$ Finally, patients with depression are vulnerable to unhealthy lifestyle behaviours such as smoking, overeating (leading to obesity, dyslipidaemia) and stressful emotional state, all of which are risk factors for coronary artery disease. ${ }^{49}$

The following several limitations should be considered in the current study. First, our study followed a crosssectional design, so no clear cause-effect conclusion could be directly drawn. In addition, in some studies, standard diagnostic interviews according to the Diagnostic and Statistical Manual of Mental Disorders, Fourth Edition criteria were performed to obtain the diagnosis of MDD ${ }^{161920}$ while in others certain diagnostic scale was used. ${ }^{161720215051}$ However, in the current study, we did not have a clinical diagnosis of MDD, although we noted that a cut-off value of 10 on PHQ-9 to screen for MDD was both highly sensitive $(85 \%)$ and specific $(89 \%) \cdot{ }^{25}$ Further studies with optimal designs are needed. In addition, the covariate data we collected were limited, and we did not have information on socioeconomic level, ${ }^{52} 53$ education level, physical activity level, dietary habits, assessment of cognitive impairment, ${ }^{54}$ and medications taken by participants as different antidepressant medications might have opposite impact on arterial stiffness, ${ }^{55}$ all of which might have influenced our results. Our study might still lack statistical power despite having a relatively large sample size $(\mathrm{n}=1334)$. For example, distinguished trends emerged among the subgroups of sex in the association between increased degree of depressive symptoms and baPWV; however, the result of the interaction was nonsignificant ( $p$ for interaction $=0.072$ ). Finally, our participants were from our health management centre, and although there were no specific restrictions for those who decided to receive health check-ups it should be noted that the costs of examinations were paid by the participants themselves. So a selection bias existed and our sample could not fully represent the general population in the real world. Therefore, extrapolation of our results to other populations should be done cautiously.

\section{CONCLUSION}

Our findings suggested that degree of depressive symptoms was independently associated with arterial stiffness, and further stratified analysis showed that subjects with depressive symptoms whose blood pressures were not optimal or complicated with diabetes mellitus were more susceptible to advanced arterial stiffness. Our results suggest that specific populations might need extra attention with regard to prevention of arterial stiffness due to the effect of depression.

Contributors Data collection: LP, TL, YZ and FL. Data analysis and interpretation: SB, XL and CZ. Manuscript writing: LP and SB. Study design: TY and CZ. Manuscript revision: $\mathrm{CZ}$. All authors approved the final version of the manuscript.
Funding This research was supported by the National Natural Science Foundation of China (31500726 and 81700279).

Competing interests None declared.

Patient consent for publication Not required.

Ethics approval Our study protocol was approved by the Ethics Committee of Xiangya Hospital, Central South University, and all procedures were conducted in accordance with ethical standards. All participants provided written informed consent.

Provenance and peer review Not commissioned; externally peer reviewed.

Data availability statement Data are available upon reasonable request. Data from the current study are available from the corresponding author ( chenglongzhang@csu.edu.cn) upon reasonable request.

Open access This is an open access article distributed in accordance with the Creative Commons Attribution Non Commercial (CC BY-NC 4.0) license, which permits others to distribute, remix, adapt, build upon this work non-commercially, and license their derivative works on different terms, provided the original work is properly cited, appropriate credit is given, any changes made indicated, and the use is non-commercial. See: http://creativecommons.org/licenses/by-nc/4.0/.

\section{ORCID iD}

Chenglong Zhang http://orcid.org/0000-0002-9656-3099

\section{REFERENCES}

1 Cao Y, Li W, Shen J, et al. Health-Related quality of life and symptom severity in Chinese patients with major depressive disorder. Asia Pac Psychiatry 2013;5:276-83.

2 Ferrari AJ, Somerville AJ, Baxter AJ, et al. Global variation in the prevalence and incidence of major depressive disorder: a systematic review of the epidemiological literature. Psychol Med 2013;43:471-81.

$3 \mathrm{Gu}$ L, Xie J, Long J, et al. Epidemiology of major depressive disorder in mainland China: a systematic review. PLoS One 2013;8:e65356.

4 González HM, Tarraf W, Whitfield KE, et al. The epidemiology of major depression and ethnicity in the United States. J Psychiatr Res 2010;44:1043-51.

5 Hare DL, Toukhsati SR, Johansson P, et al. Depression and cardiovascular disease: a clinical review. Eur Heart $J$ 2014;35:1365-72.

6 Li Z, Li Y, Chen L, et al. Prevalence of depression in patients with hypertension: a systematic review and meta-analysis. Medicine 2015;94:e1317

7 Rutledge T, Reis VA, Linke SE, et al. Depression in heart failure a meta-analytic review of prevalence, intervention effects, and associations with clinical outcomes. J Am Coll Cardiol 2006;48:1527-37.

8 Osler M, Mårtensson S, Wium-Andersen IK, et al. Depression after first hospital admission for acute coronary syndrome: a study of time of onset and impact on survival. Am J Epidemiol 2016;183:218-26.

9 Lichtman JH, Froelicher ES, Blumenthal JA, et al. Depression as a risk factor for poor prognosis among patients with acute coronary syndrome: systematic review and recommendations: a scientific statement from the American heart association. Circulation 2014;129:1350-69.

10 González HM, Tarraf W. Comorbid cardiovascular disease and major depression among ethnic and racial groups in the United States. Int Psychogeriatr 2013;25:833-41.

11 Tomiyama $\mathrm{H}$. Arterial stiffness: is this a marker for the current status or future? J Atheroscler Thromb 2016;23:1026-7.

12 Williams B, Mancia G, Spiering W, et al. 2018 ESC/ESH guidelines for the management of arterial hypertension. Eur Heart $J$ 2018;39:3021-104.

13 Ben-Shlomo Y, Spears M, Boustred C, et al. Aortic pulse wave velocity improves cardiovascular event prediction: an individual participant meta-analysis of prospective observational data from 17,635 subjects. J Am Coll Cardiol 2014;63:636-46.

14 Ohkuma T, Ninomiya T, Tomiyama H, et al. Brachial-Ankle pulse wave velocity and the risk prediction of cardiovascular disease: an individual participant data meta-analysis. Hypertension 2017:69:1045-52.

15 Vlachopoulos C, Aznaouridis K, Terentes-Printzios D, et al. Prediction of cardiovascular events and all-cause mortality with brachial-ankle elasticity index: a systematic review and meta-analysis. Hypertension 2012;60:556-62. 
16 Tiemeier H, Breteler MMB, van Popele NM, et al. Late-Life depression is associated with arterial stiffness: a population-based study. J Am Geriatr Soc 2003;51:1105-10.

17 van Sloten TT, Mitchell GF, Sigurdsson S, et al. Associations between arterial stiffness, depressive symptoms and cerebral small vessel disease: cross-sectional findings from the AGES-Reykjavik study. $J$ Psychiatry Neurosci 2016;41:162-8.

18 Dietz LJ, Matthews KA. Depressive symptoms and subclinical markers of cardiovascular disease in adolescents. J Adolesc Health 2011;48:579-84.

19 Seldenrijk A, van Hout HPJ, van Marwijk HWJ, et al. Depression, anxiety, and arterial stiffness. Biol Psychiatry 2011;69:795-803.

20 Onete V, Henry RM, Sep SJS, et al. Arterial stiffness is associated with depression in middle-aged men - the Maastricht Study. $J$ Psychiatry Neurosci 2018;43:111-9.

21 Lewis TT, Sutton-Tyrrell K, Penninx BW, et al. Race, psychosocial factors, and aortic pulse wave velocity: the health, aging, and body composition study. J Gerontol A Biol Sci Med Sci 2010;65:1079-85.

22 Seldenrijk A, van Hout HPJ, van Marwijk HWJ, et al. Sensitivity to depression or anxiety and subclinical cardiovascular disease. $J$ Affect Disord 2013;146:126-31.

23 Committee for the Korean Guidelines for the Management of Dyslipidemia. 2015 Korean guidelines for the management of dyslipidemia: Executive summary (English translation). Korean Circ J 2016;46:275-306.

24 Kroenke K, Spitzer RL, Williams JB. The PHQ-9: validity of a brief depression severity measure. J Gen Intern Med 2001;16:606-13.

25 Manea L, Gilbody S, McMillan D. Optimal cut-off score for diagnosing depression with the patient health questionnaire (PHQ-9): a meta-analysis. CMAJ 2012;184:E191-6.

26 Salk RH, Hyde JS, Abramson LY. Gender differences in depression in representative national samples: meta-analyses of diagnoses and symptoms. Psychol Bull 2017;143:783-822.

27 Schuch JJJ, Roest AM, Nolen WA, et al. Gender differences in major depressive disorder: results from the Netherlands study of depression and anxiety. J Affect Disord 2014;156:156-63.

28 Vermeersch SJ, Rietzschel ER, De Buyzere ML, et al. Age and gender related patterns in carotid-femoral PWV and carotid and femoral stiffness in a large healthy, middle-aged population. $J$ Hypertens 2008;26:1411-9.

29 Schram MT, Sep SJS, van der Kallen CJ, et al. The Maastricht study: an extensive phenotyping study on determinants of type 2 diabetes, its complications and its comorbidities. Eur J Epidemio 2014;29:439-51.

30 Yambe M, Tomiyama H, Yamada J, et al. Arterial stiffness and progression to hypertension in Japanese male subjects with high normal blood pressure. J Hypertens 2007;25:87-93.

31 AlGhatrif M, Strait JB, Morrell CH, et al. Longitudinal trajectories of arterial stiffness and the role of blood pressure: the Baltimore longitudinal study of aging. Hypertension 2013;62:934-41.

32 Godin O, Elbejjani M, Kaufman JS. Body mass index, blood pressure, and risk of depression in the elderly: a marginal structural model. Am J Epidemiol 2012;176:204-13.

33 Zimmerman JA, Mast BT, Miles T, et al. Vascular risk and depression in the Hispanic established population for the epidemiologic study of the elderly (EPESE). Int J Geriatr Psychiatry 2009;24:409-16.

34 Hildrum B, Mykletun A, Holmen J, et al. Effect of anxiety and depression on blood pressure: 11-year longitudinal population study. Br J Psychiatry 2008;193:108-13.

35 Scuteri A, Spalletta G, Cangelosi M, et al. Decreased nocturnal systolic blood pressure fall in older subjects with depression. Aging Clin Exp Res 2009;21:292-7.

36 Scuteri A, Morrell CH, Orru' M, et al. Gender specific profiles of white coat and masked hypertension impacts on arterial structure and function in the Sardinia study. Int J Cardiol 2016;217:92-8.
37 Scuteri A, Castello L, Coluccia R, et al. Depression is associated with increased occurrence of left ventricle concentric geometry in older subjects independently of blood pressure levels. Nutr Metab Cardiovasc Dis 2011;21:915-21.

$38 \mathrm{Knol}$ MJ, Twisk JWR, Beekman ATF, et al. Depression as a risk factor for the onset of type 2 diabetes mellitus. A meta-analysis. Diabetologia 2006;49:837-45.

39 Nouwen A, Winkley K, Twisk J, et al. Type 2 diabetes mellitus as a risk factor for the onset of depression: a systematic review and metaanalysis. Diabetologia 2010;53:2480-6.

40 Samaan Z, Garasia S, Gerstein HC, et al. Lack of association between type 2 diabetes and major depression: epidemiologic and genetic evidence in a multiethnic population. Transl Psychiatry 2015;5:e618.

41 Berk M, Williams LJ, Jacka FN, et al. So depression is an inflammatory disease, but where does the inflammation come from? BMC Med 2013;11:200.

42 Slavich GM, Irwin MR. From stress to inflammation and major depressive disorder: a social signal transduction theory of depression. Psychol Bull 2014;140:774-815.

43 van Dooren FEP, Schram MT, Schalkwijk CG, et al. Associations of low grade inflammation and endothelial dysfunction with depression - The Maastricht Study. Brain Behav Immun 2016;56:390-6.

44 Lopez-Vilchez I, Diaz-Ricart M, Navarro V, et al. Endothelial damage in major depression patients is modulated by SSRI treatment, as demonstrated by circulating biomarkers and an in vitro cell model. TransI Psychiatry 2016;6:e886.

45 Won E, Kim Y-K, Stress KYK. Stress, the autonomic nervous system, and the Immune-kynurenine pathway in the etiology of depression. Curr Neuropharmacol 2016;14:665-73.

46 Wang S-Y, Duan K-M, Tan X-F, et al. Genetic variants of the kynurenine-3-monooxygenase and postpartum depressive symptoms after cesarean section in Chinese women. J Affect Disord 2017;215:94-101.

47 Whelton SP, Blankstein $\mathrm{R}$, Al-Mallah $\mathrm{MH}$, et al. Association of resting heart rate with carotid and aortic arterial stiffness: multi-ethnic study of atherosclerosis. Hypertension 2013;62:477-84.

48 Jaiswal M, Urbina EM, Wadwa RP, et al. Reduced heart rate variability is associated with increased arterial stiffness in youth with type 1 diabetes: the search CVD study. Diabetes Care 2013;36:2351-8.

49 Katon WJ. Clinical and health services relationships between major depression, depressive symptoms, and general medical illness. Biol Psychiatry 2003;54:216-26.

50 van Sloten TT, Boutouyrie P, Tafflet M, et al. Carotid artery stiffness and incident depressive symptoms: the Paris prospective study III. Biol Psychiatry 2019;85:498-505

51 Lin I-M, Lu H-C, Chu C-S, et al. The relationship between Brachial-Ankle pulse wave velocity and depressive symptoms among patients with coronary artery disease. Acta Cardiol Sin 2017;33:303-9.

52 Wiernik E, Lemogne C, Thomas F, et al. Perceived stress, common carotid intima media thickness and occupational status: the Paris prospective study III. Int J Cardiol 2016;221:1025-30.

53 Cho Y, Lim TH, Kang H, et al. Socioeconomic status and depression as combined risk factors for acute myocardial infarction and stroke: a population-based study of 2.7 million Korean adults. J Psychosom Res 2019;121:14-23.

54 Scuteri A, Wang $\mathrm{H}$. Pulse wave velocity as a marker of cognitive impairment in the elderly. J Alzheimers Dis 2014;42 Suppl 4:S401-10.

55 Scuteri A, Modestino A, Fedullo F, et al. Depression treatment selectively modifies arterial stiffness in older participants. J Gerontol A Biol Sci Med Sci 2013;68:719-25. 